\title{
Effect of defects on thermal denaturation of DNA oligomers
}

\author{
Navin Singh and Yashwant Singh \\ Department of Physics, Banaras Hindu University, \\ Varanasi 221005 , India
}

\begin{abstract}
The effect of defects on the melting profile of short heterogeneous DNA chains are calculated using the Peyrard-Bishop Hamiltonian. The on-site potential on a defect site is represented by a potential which has only the short-range repulsion and the flat part without well of the Morse potential. The stacking energy between the two neigbouring pairs involving a defect site is also modified. The results are found to be in good agreement with the experiments.
\end{abstract}

87.10+e, 63.70.+h, 64.70.-p

Typeset using REVTEX 
DNA thermal denaturation is the process of separating the two strands wound in a double helix into two single strands upon heating. Several experiments [1,2] on dilute DNA solutions have provided evidence for the existence of a thermally driven melting transition corresponding to the sudden opening of base pairs at a critical(or melting) temperature $T_{m}$. At $T_{m}$ one-half of the DNA denatured. The understanding of this remarkable one-dimensional cooperative phenomena in terms of standard statistical mechanics, i.e., a Hamiltonian model with temperature independent parameters is a subject of current interest [3 8 ].

DNA is modelled as a quasi one dimensional lattice composed of $\mathrm{N}$ base pair units [9]. The double helix is held together by hydrogen bonds between complementary bases on opposite strands and hydrophobic or stacking interactions between nearest neighbor bases on opposite strands. Each base pair is in one of the two states; either open (non-hydrogen) or intact (hydrogen-bonded). A Hamiltonian model which has been found appropriate to represent interactions in DNA is the Peyrard-Bishop (PB) model [3, [4]. The PB model is written as,

$$
H=\sum_{n}\left[\frac{p_{n}^{2}}{2 m}+W\left(y_{n}, y_{n+1}\right)+V_{n}\left(y_{n}\right)\right]
$$

where $m$ is the reduced mass of a base pair, $y_{n}$ denotes the stretching of the hydrogen bonds connecting the two bases of the $n^{\text {th }}$ pair and

$$
p_{n}=m\left(\frac{d y_{n}}{d t}\right)
$$

The on site potential $V_{n}\left(y_{n}\right)$ describes the interaction of the two bases of the $n^{\text {th }}$ pair. The Morse potential,

$$
V_{n}\left(y_{n}\right)=D_{n}\left(e^{-a y_{n}}-1\right)^{2}
$$

which is usually taken to represent the on-site interaction represents not only the $\mathrm{H}$ bonds connecting two bases belonging to opposite strands, but also the repulsive interactions of the phosphates and the surrounding solvents effects. The flat part at large values of the displacement of this potential emulates the tendency of the pair "melt" at high temperatures 
as thermal phonons drive the nucleotides outside the well (see Fig.1) and towards the flat portion of the potential.

The stacking energy between the two neighboring base pairs is described by the anharmonic potential

$$
W\left(y_{n}, y_{n+1}\right)=\frac{k}{2}\left[1+\rho e^{-\alpha\left(y_{n}+y_{n+1}\right)}\right]\left(y_{n}-y_{n-1}\right)^{2}
$$

The choice of this form of $W\left(y_{n}, y_{n-1}\right)$ has been motivated by the observation that the stacking energy is not a property of individual bases but a character of the base pair, themselves [7]. When due to stretching the hydrogen bonds connecting the bases break, the electronic distribution on bases is modified causing the stacking interaction with adjacent bases to decrease. This is taken into account by the exponential term in Eq.(3). One may note that the effective coupling constant decreases from $k(1+\rho)$ to $k$ when either one of the two interacting base pairs is stretched. Eq.(3), therefore, takes care of changes in stacking energy due to breaking of hydrogen bonds in base pairs due to stretching. This decrease in coupling provides a large entropy in the denaturation. The parameter $\alpha$ in Eqs.(3) defines the "anharmonic range".

The model Hamiltonian of Eq.(1) has extensively been used to study the melting profile of a very long $(N \rightarrow \infty)$ and homogeneous DNA chain using both statistical mechanical calculations and the constrained temperature molecular dynamics [10,11]. Analytical investigation of nonlinear dynamics of the model suggests that intrinsic energy localization can initiate the denaturation [12]. In case of long homogeneous DNA chain the model exhibits a peculiar type of first-order transition with finite "melting entropy", a discontinuity in the fraction of bound pairs and divergent correlation lengths. However, as the value of the stacking parameter $\alpha$ increases and the range of the "entropy barrier" becomes shorter than or comparable to the range of the Morse potential the transition changes to second order. The crossover is seen at $\alpha / a=0.5[8]$.

When one considers chain having inhomogeneity in base pair sequence, one faces a problem in investigating its statistical mechanics as the transfer-integral method which has been 
used in the case of homogeneous chain is no longer valid. Attempts have, however, been made to use the model Hamiltonian of Eq.(1) for heterogeneous chains either by modelling the heterogeneity with quenched disorder [6] or by properly choosing basis sets of orthonormal functions for the kernels appearing in the expansion of the partition function [7].

In this note we investigate the effect of defects on the melting profile of short DNA chains of heterogeneous compositions. A defect on DNA chain means a mismatched basepair. For example, if one strand of DNA has adenine on a site the other strand has guanine or cytosine instead of thymine on the same site. In such a situation the pair will remain in open state at all temperatures as two nucleotides cannot join each other through hydrogen bonds. Oligonucleotide probes are commonly used to identify the presence of unrelated nucleic acids. In this context it is therefore important to discriminate the targets that differ from one another by a single or more nucleotides.

Since the partition function of the PB model is convergent only in the limit of infinite number of base-pairs $\mathrm{N}$ (i.e. $N \rightarrow \infty)$ 《\#, it is therefore, necessary in the case of short chains to consider not only the breaking of hydrogen bonds between single base pair, but also the complete dissociation of the two strands forming the double helix. In other words, in the case of short chains there is a need to consider thermal equilibrium between dissociated strands and associated double strands(the duplex) and a thermal equilibrium in the duplexes, between broken and unbroken interbase hydrogen bonds.

The average fraction $\theta$ of bonded base pairs can be factored as $\theta=\theta_{\text {ext }} \theta_{\text {int }}$ [7, [13]. $\theta_{\text {ext }}$ is the average fraction of strands forming duplexes, while $\theta_{\text {int }}$ is the average fraction of unbroken bonds in the duplexes. The equilibrium dissociation of the duplex $C_{2}$ to single strand $C_{1}$ may be represented by the relation $C_{2} \rightleftharpoons 2 C_{1}$ [8,9]. The dissociation equilibrium can be neglected in the case of long chains; while $\theta_{\text {int }}$ and thus $\theta$ go to zero when $\theta_{\text {ext }}$ is still practically 1 . This is because in the case of long DNA fragments when $\theta$ goes practically from 1 to 0 at the melting transition, the two strands may not get completely separated; while most bonds are disrupted and the DNA has denatured, few bonds still remaining prevent the two strands from going apart each other. It is only at $T>>T_{m}$ there will be 
a real separation. Therefore, at the transition the double strand is always a single molecule and in calculation based on the PB model one has to calculate only $\theta_{\text {int }}$. On the contrary, in the case of short chains the process of single bond disruption and strand dissociation tend to happen in the same temperature range; therefore, the computation of both $\theta_{\text {int }}$ and $\theta_{\text {ext }}$ is essential.

For the computation of $\theta_{\text {int }}$ one has to separate the configurations describing a double strand on the one hand, and dissociated single strand on the other. For this we follow the method suggested by Campa and Giansanti [13]. The $n^{\text {th }}$ bond is considered open if the value of $y_{n}$ is larger than a chosen threshold $y_{0}$. A configuration belongs to the double strands if at least one of the $y_{n}^{\prime s}$ is smaller than $y_{0}$. One can therefore define $\theta_{\text {int }}$ for an $\mathrm{N}$ base pair duplexes by:

$$
\theta_{i n t}=\frac{1}{N} \sum_{i=1}^{i=N}\left\langle\vartheta\left(y_{0}-y_{i}\right)\right\rangle
$$

where $\vartheta(y)$ is Heaviside step function and the canonical average $\langle$.$\rangle is defined considering$ only the double strand configurations. For $y_{0}$, we have taken a value of $2 \stackrel{\circ}{A}$. Since the PB model couples only the nearest neighbors the calculation of canonical averages in Eqs.(4) reduced to multiplication of finite matrices. The discretization of the coordinate variables and introduction of a proper cutoff on the maximum value of $y^{\prime s}$ [10] determines the size of the matrices and the number of base pairs in the chain the number of matrices to be multiplied.

Since we are concerned with a heterogenous DNA chain, we have selected two different values of $D_{n}$ (see Eq. 2) according to the two possible base pairs; adenine-thymine (A$\mathrm{T}$ ) and guanine-cytosine (G-C). While A-T has the two hydrogen bonds, the G-C pair has three. Because of this $D_{n}$ for G-C bonds is chosen as nearly 1.5 times larger than the one representing to the A-T bonds. The complete set of values in our calculations are: $D_{A T}=$ $0.05 \mathrm{eV}, \quad D_{G C}=0.075 \mathrm{eV}, \quad a_{A T}=4.2 \stackrel{\circ}{A^{-1}}, \quad a_{G C}=6.9, \quad \stackrel{\circ}{A}^{-1} k=0.025 \mathrm{eV} / \stackrel{\circ}{A}^{2}, \quad \rho=$ 2, and $\alpha=0.35 \stackrel{\circ}{A}-1$

Since at a defect site the nucleotides of the two strands do not associate themselves 
through hydrogen bonds we replace the on-site Morse potential by a potential shown in Fig. 1 by full line.

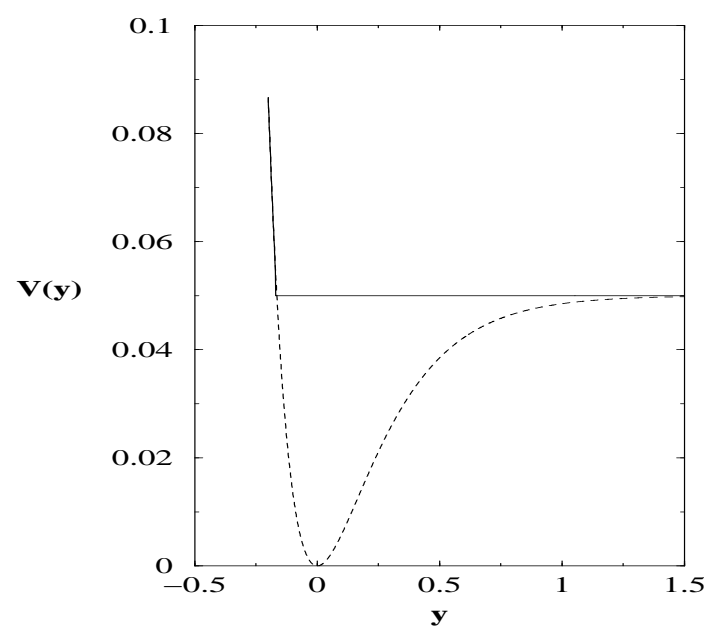

Fig. 1 The on-site potential $\mathrm{V}(\mathrm{y})$ as a function of displacements. The dotted line represents the Morse potential (see Eqn.(2) and the full line the potential chosen to represent the interaction at the defect sites.

This potential has repulsive part as well as the flat part of the Morse potential but not the well which arises due to hydrogen bonding interactions. Due to defect on a site the stacking interactions with adjacent bases will also be affected. As argued above, the formation of hydrogen bonds changes the electronic distribution on base pairs causing stronger stacking interactions with adjacent bases. Therefore, when one of the base pairs without hydrogen bonds is involved the stacking interaction will be weaker compared to the case when both base pairs are in intact state. This fact has been taken into account in our calculation by reducing the anharmonicity coefficient $\rho$ to its half value wherever the defective site appeared in Eq.(3).

For $\theta_{\text {ext }}$ we use the expression given in Ref. [13]. Thus,

$$
\theta_{\text {ext }}=1+\delta-\sqrt{\delta^{2}+2 \delta}
$$

where

$$
\delta=\frac{Z(A) Z(B)}{2 N_{0} Z(A B)}
$$


Here $\mathrm{Z}(\mathrm{A}), \mathrm{Z}(\mathrm{B})$ and $\mathrm{Z}(\mathrm{AB})$ are the configurational isothermal- isobaric partition functions of systems consisting of molecular species A, B and AB respectively. $N_{j}$ is the number of molecules of species $j$ in volume $\mathrm{V}$ and $2 N_{0}=2 N_{A B}+N_{A}+N_{B}$. In deriving Eq.(6) $N_{A}$ has been taken equal to $N_{B}$ and $\theta_{\text {ext }}$ defined as

$$
\theta_{e x t}=\frac{N_{A B}}{N_{0}}
$$

The partition function for each species can be factored into internal and external part and can be written as [13],

$$
\frac{Z(A) Z(B)}{2 N_{0} Z(A B)}=\frac{Z_{i n t}(A) Z_{i n t}(B)}{a_{a v} Z_{i n t}(A B)} \frac{a_{a v} Z_{e x t}(A) Z_{e x t}(B)}{2 N_{0} Z_{e x t}(A B)}
$$

where $a_{a v}=\sqrt{a_{A T} a_{G C}}$

In analogy to what has been proposed for the Ising model [3] on the basis of partition function of rigid molecules [16], one makes the following choice

$$
\frac{a_{a v} \cdot Z_{e x t}(A) Z_{e x t}(B)}{2 N_{0} Z_{e x t}(A B)}=\frac{n^{*}}{n_{0}} N^{-p \theta_{i n t}+q}
$$

where $n^{*}$ is a chosen reference concentration as $1 \mu M$ while $n_{0}$ is the single strand concentration which we have chosen as $3.1 \mu M . p$ and $q$ are the parameters which can be calculated using experimental results.

We have considered the following two different oligonucleotides with sequence given by:

$$
\begin{aligned}
& \text { (A) }{ }^{5^{\prime}} G T G T T A A C G T G A G T A T A G C G T_{3^{\prime}} \\
& \text { (B) }{ }^{{ }^{\prime}} G G T_{11} G G_{3^{\prime}}
\end{aligned}
$$

and by the respective complementary strands when there is no defect. The melting profile of oligonucleotides (A) has been calculated by Campa and Giansanti 13. They found very good agreement with experiment. We have extended their calculation and have studied the effect of defects on the melting profile. The results are plotted in Fig. (2). Our results for defectless chain agree very well with that given in refs. [13] and with experiments [14. 


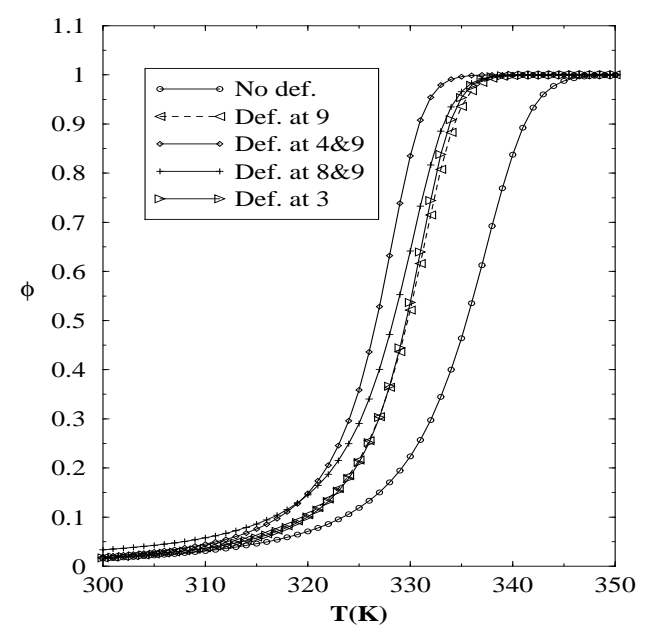

Fig. 2 Plot showing the variation of melting temperature with defect. Here $p=29.49$ and $q=27.69$

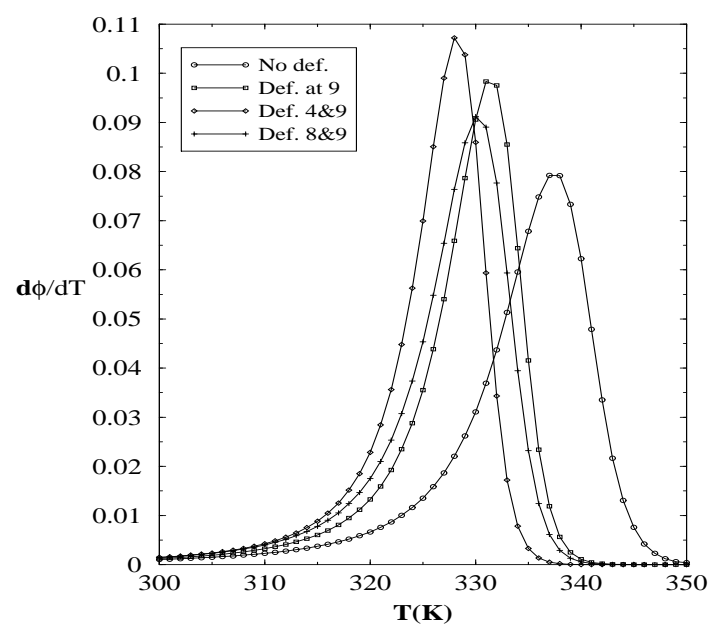

Fig.3 Plot showing the differential melting curve

We used the same parameters except the modification as stated above in the Morse potential and the anharmonic stacking potential term to calculate the melting profile with one defect on a site. Our calculation indicates that the melting profile of the chain does not depend on the location of the defect site. When we introduced two defects one at $4^{\text {th }}$ and the other at $9^{\text {th }}$ sites we found the melting curve shifts to further lower temperature. While we found the melting temperature $T_{m}$ decreases from $335.7 \mathrm{~K}$ to nearly $329.7 \mathrm{~K}$ (i.e. $\left.\Delta T_{m}^{(0,1)} \sim 6 K\right)$ in the presence of one defect, the decrease in $T_{m}$ from one to two defects is 
only $\Delta T_{m}^{(1,2)} \sim 3 K$. These results are in good agreement with the experimental observations [14. We also put the two defects at the consecutive sites;i.e., at $8^{\text {th }}$ and $9^{\text {th }}$ sites. As Fig. 2 shows there is a change in the melting profile compared to the case when the two defect sites were apart. This change is more clearly seen in the plot of $d \phi / d T$ shown in Fig.3.

The oligonucleotides (B) has been studied by Bonnet et al [15]. They measured the change in the melting temperature with one defect placed on different sites of the chain. They found $T_{m}=315 \mathrm{~K}$ for defectless chain and $\Delta T_{m}=8 \mathrm{~K}$ with one defect. Their results shows that the location of the defect on the chain has practically no effect on the melting temperature of the chain in agreement with our calculations.

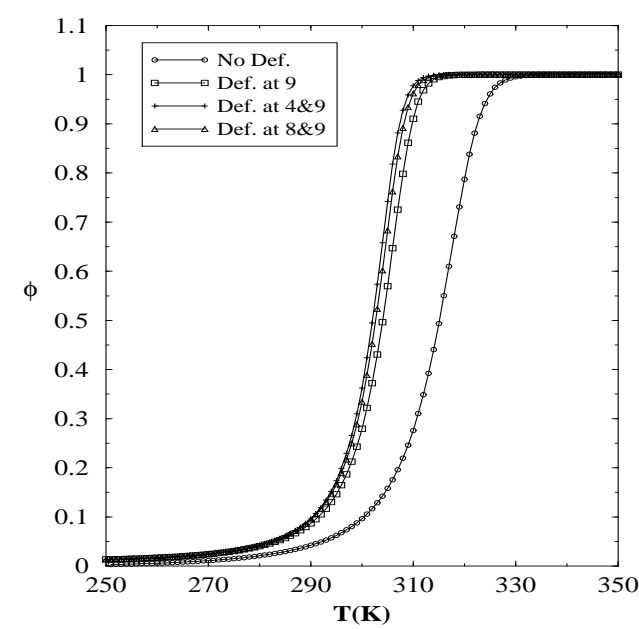

Fig. 4 Plot showing the variation of melting temperature with defect. Here $p=34.46$ and $q=32.45$ 


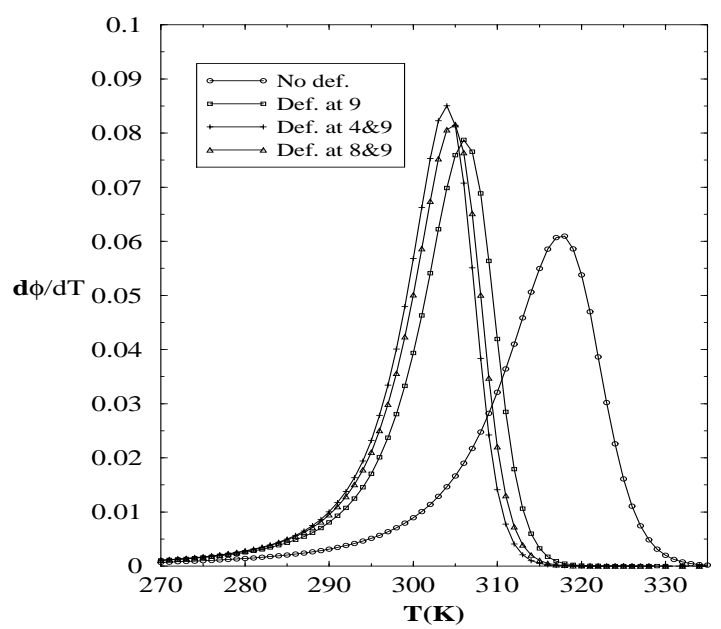

Fig. 5 Plot showing differential melting curves

We plot our results in Fig. 4. The force parameters are same as given in Eq.(5). Since the experimental conditions are slightly different than the one for chain (A) we adjusted parameters $p$ and $q$ so that melting profile for the defect less chain agrees with the experimental result. Using these values of the parameters we have calculated the melting profile with one and two defects. We find that while $\Delta T_{m}^{(0,1)} \sim 10 K$ and $\Delta T_{m}^{(1,2)} \sim 3 K$. Fig. 5 shows the plot of $d \phi / d T$.

In conclusion we wish to emphasize that our calculations show that the PB model is capable of describing the melting profile of oligonucleotides of heterogeneous composition in the presence of defects also.

This work was supported by the Department of Science and Technology, (India) through research grant. 


\section{REFERENCES}

[1] W. Saenger, Principles of Nucleic Acid Structures, ( Springer-Verlag, New York 1984)

[2] L. Stryer, Biochemistry, (W.H. Freeman and Company, New York 1995)

[3] M. Peyrard and A. R. Bishop, Phys. Rev. Lett. 62 2755(1989)

[4] T. Dauxois, M. Peyrard and A. R. Bishop, Phys. Rev. E 47684 (1993)

[5] E. W. Prohofsky, Statistical Mechanics and Stability of Macromolecules ( Cambridge University Press, Cambridge 1995)

[6] D. Cule and T. Hwa, Phys. Rev. Lett. 79, 2375(1997)

[7] Y. Zhang, W. M. Zheng, J. X. Liu and Y. Z. Chen, Phys. Rev. E 56, 7100 (1997)

[8] N. Theodorakopoulos, T. Dauxois and M. Peyrard, Phys. Rev. Lett.85, 6(2000)

[9] R.M. Wartell and A.S. Benight, Phys. Rep. 126, 67(1985).

[10] T. Dauxois and M. Peyrard, Phys. Rev. E 514027 (1995)

[11] T. Dauxois, M. Peyrard and A. R. Bishop, Phys. Rev. E 47 R44 (1993)

[12] T. Dauxois and M. Peyrard, Phys. Rev. Lett. 703915 (1993)

[13] A. Campa and A. Giansanti, Phys. Rev. E 58, 3585(1998)

[14] A. Bonincontro,M. Matzeu, F.Mazzei, A. Minoprio and F. Pedone, Biochim. Biophys. Acta 1171, 288(1993)

[15] G. Bonnet, Sanjay Tyagi, A. Libchaber and F.R. Kramer Proc. of Natl. Acad. Sci., USA, 96, 6171(1999)

[16] L.D. Landau and E.M. Lifshitz, Statistical Physics (Oxford Pergamon Press, 1980) 